\title{
Augmented Reality Electronic Glasses Prototype to Improve Vision in Older Adults
}

\author{
Lilian Ocares Cunyarachi ${ }^{1}$ \\ Facultad de Ciencias e Ingeniería \\ Universidad de Ciencias y Humanidades
}

\author{
Alexandra Santisteban Santisteban ${ }^{2}$ \\ Facultad de Ciencias e Ingenieria \\ Universidad de Ciencias y Humanidades
}

\author{
Laberiano Andrade-Arenas ${ }^{3}$ \\ Facultad de Ciencias e Ingenieria \\ Universidad de ciencias y Humanidades
}

\begin{abstract}
In this article, we focus on the elderly who suffer from low vision. We seek to design Augmented reality electronic glasses to help the elderly who suffer from vision problems, which causes a limitation when performing their daily activities, so this can affect their development in society causing serious physical and emotional damage, so we used a set of scientific articles that analyzed the percentage of visual impairment. Technology has demonstrated on numerous occasions that it can be a great ally for the health and well-being of the elderly. In this work, the objective is to design electronic glasses to help the elderly improve their vision. The methodology used is Design Thinking, which thanks to the phases of this methodology helps us to understand, collect information about the problem and give a solution, the result obtained is a prototype of electronic glasses in which it will benefit adults who suffer from low vision. As for the case study, we will show the design of the mobile application and the detailed development of the prototype.
\end{abstract}

Keywords-Augmented reality; design thinking; electronic glasses; low vision; seniors

\section{INTRODUCTION}

The present research refers to the benefits of electronic glasses in elderly people, in the branch of ophthalmology as well. The low vision problems are a limitation of the visual capacity that seriously affects people, consequently it prevents the patients to be able to carry out their daily activities in such a way that they do not manage to interact with the society [1].

The importance of research is to design electronic glasses, in order to improve the vision of people who suffer from problems of eye loss so that we can solve the problems of vision in older adults, consequently over the years suffer eye damage which does not allow them to see clearly, so these glasses will help people to be able to have better near vision [2]. This way the electronic glasses have implemented a camera with high resolution hd generating great luminosity that manages to capture the environment and all that where the person directs the intelligent glasses, in such a way that the image managed to process an algorithm in which it was adjusted and modified to detail each one of the images identifying separately according to the degree of affection of each one of them [3]. To close the idea the advantage of these electronic glasses will allow to project videos and to take pictures that are received through a connection via bluetooth or wifi of the connector HDMI that comes incorporated [4]. According to the World Health Organization [5], the estimated number of people with this visual disability is 253 million, of which 36 million are blind and 217 million have moderate-severe visual impairment [6]. It is important to note that $81 \%$ of people with blindness and visual disability are over 50 years old, which confirms that the vast majority of people who suffer from vision loss are adults [7].It is important to note that a detailed study was conducted in Peru, through a National Specialized Survey on Disability (Enedis). It was determined that the highest disability is in the visual limitation with a 48.3\% [8]. As a result, the WHO and Enedis statistics on visual disability in Peru were known, with a high percentage of the elderly population [9].

The objective of our work is to be able to help older people with low vision by designing electronic augmented reality glasses that allow the capture of high quality images in real time, to improve visual perception and thus daily autonomy.

This paper is structured as follows: Section II will describe in detail the methodology used for the design. Section III will show the case study, Section IV will show the results and discussion and finally, Section V will present the conclusions.

\section{Methodology}

The methodology to be used will be Design Thinking, since it is a process originally oriented to the creation or improvement of products, which allows us to solve the problems reducing the risks and in such a way that increases the possibilities of success, focusing on the needs of the user, from then on the information about the topic or problem is understood and collected, then it is observed, the point of view of the team is given, a solution is devised, prototypes are made and finally the test in such a way that it is possible to connect the knowledge of different disciplines to arrive at a technically viable and economically profitable solution [10]. Design Thinking is an agile methodology for digital innovation, in such a way that it extracts the benefits of one and other methods that complement the challenges posed. The conformation of the concept to the agile evolution opens better opportunities and can be used in different agile iterations [11]. In Fig. 1 both the problem space and the solution space are mentioned, in the same way there is space to explore multiple options through the divergent phase. 


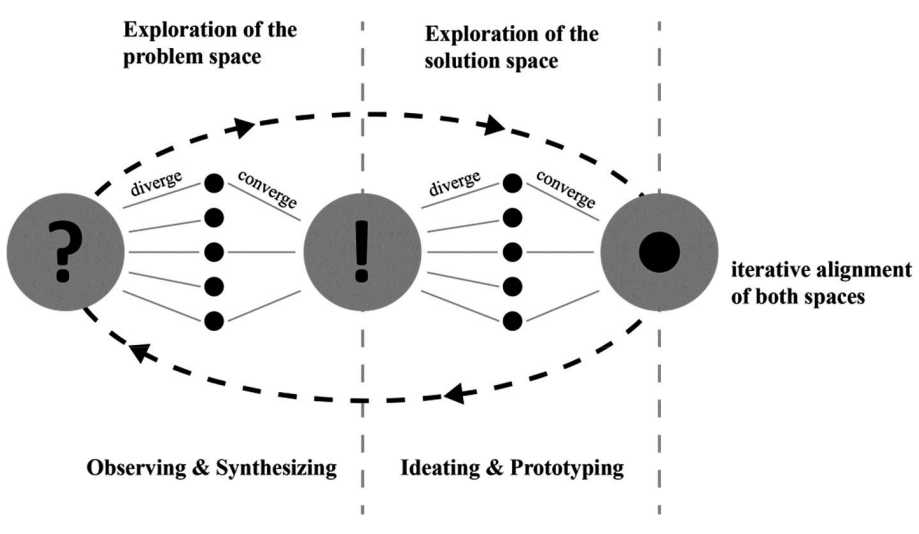

Fig. 1. Problem and Solution Space in Design Thinking

\section{Phases of Design Thinking}

1) Understand: The first phase is to understand, collect and analyze the information about the topic or problem to be solved, in order to understand the life of the users, as well as the different problems and needs they have.

2) Observe: The second phase collects and processes information on user needs through client interviews and the research.

3) Point of View: The third phase collects the information from the first two phases that is shared at the meeting and becomes a framework called a point of view.

4) Ideate: In the fourth phase, the development of an interdisciplinary team crosses through different processes that have been observed in detail and from then on a solution is designed with opportunities for change during implementation.

5) Prototype: This is the central phase of Design Thinking, a prototype is designed that is converted with ideas into action. The purpose of this phase is to convert the idea or solution into a digital or physical prototype.

6) Test: The last phase of the test is a thorough evaluation of the requirements and needs of the user. All these processes are highly iterative and can be repeated from any phase in which a completed product is delivered.

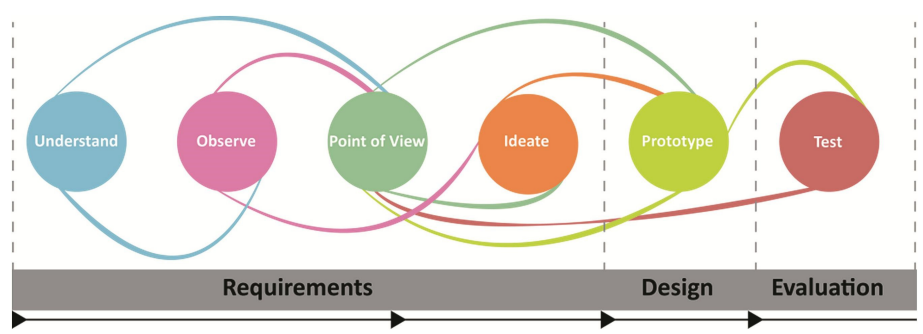

Fig. 2. Adapted Design Thinking Process Model
Fig. 2 refers to the proposed framework, the six processes of Design Thinking is adapted in the three phases as one of them is the requirement, Design and Evaluation. In the first four phases of Design Thinking, the focus is on gathering the necessary information to start a real implementation.

\section{CASe STUdy}

With the methodology mentioned below, the processes will be explained Detailed according to the phases, for which electronic glasses will be designed to help older adults with low vision.

1) Understand: As a first step, the problem will be identified by adding a fact that globally, it is estimated that approximately 1.3 billion people live with some form of low vision at distance or near. With respect to distance vision, 188.5 million people have a moderate visual impairment, 217 million have a moderate to severe visual impairment and 36 million are blind [12]. On the other hand, 826 million people have an impairment of near vision [13].The vision problems in the older adults are frequent and the consequences that can be generated, arriving to affect seriously the vision, since it loses the capacity of visualization by different reasons as a result of the cataracts, glaucoma, presbyopia, macular degeneration, among others, consequently they affect the visual health which generates difficulties that cause in their states of spirit when being limited by the ocular problems. Electronic glasses make it easier for people to comfortably perform common multi-distance tasks such as reading, watching TV, doing crafts, interacting with people, viewing posters while moving around and much more [14].

2) Observe: As a second phase we collect information on the needs in older adults and on the visual pathological diseases that are mostly lacking in older people [15] that we will mention below:

Cataract: It is defined as an opacity in the lens and is a major cause of visual loss. The lens begins to deteriorate at 40 to 45 years losing its ability to accommodate what is known as presbyopia or tired eyesight, affecting virtually the entire population and requiring the use of glasses to focus on close objects [16].

Macular Degeneration: It is a frequent cause of severe and irreversible decrease in vision in elderly patients. The disease causes lesions in the central portion of the retina and in the macula which is responsible for the central vision necessary for reading or driving [17].

Glaucoma: Glaucoma is an irreversible disease of the optic nerve that is produced by an increase in intraocular pressure. The incidence of glaucoma increases with age and is therefore a risk factor for the disease, as are high myopia and the presence of a family history of glaucoma [18].

Palpebral Ptosis: In the third age, the musculature is losing tone and sometimes it generates a sagging of the eyelids that 
can affect the vision [19].

Ocular Dryness: With age, it is common for the mucous membranes to dry out and this occurs with the lacrimal duct, causing problems of ocular dryness that can be very annoying and cause complications such as blepharitis, when the Meibomio glands of the eyelashes become obstructed, causing inflammation and oily secretion that can become infected. The treatment requires a daily and specific palpebral hygiene according to the affectation [20].

3) Point of View: From a general point of view, to address the relationship of ICT with adults we will use two perspectives: as active users who use them to do tasks of all kinds, like the rest of the population or as users receiving services to support their daily lives and thus provide the same opportunities to enjoy the benefits of electronic glasses and improve their lifestyle.

4) Ideate: The step to this phase begins by creating solutions to the problems found as we have been describing in the previous phases. We focus on our efforts to be able to understand and precise the information obtained. Next, we will present tools that will help us solve the needs and desires of the user.

Android Studio: It is a specific role for the development of applications, is a software in which provides different tools and services for different users to create and innovate with new applications, starting from the code to the design of the user interface. This tool is very simple for that reason facilitates users to understand the operation quickly. In this development environment we will find the necessary tools to create applications. It should be noted that Google revealed the new environment for the development of Android Studio app, one of the key parts of Android is its powerful code editor that is included with integrated elements such as Smart Editing ie provides a more decipherable code [21].

5) Prototype: In this phase we will show the ideal conceptual design of the glasses based on indicators with integral components as shown in Fig. 3. The screen mounted on top of the glasses, also includes peripheral components of the mounted camera, with a global positioning system (GPS) tracker, gyroscope, step detection sensors and Bluetooth headset. A rechargeable battery provides electrical power to the device. The Bluetooth headset facilitates the communication mechanism between the remote caregiver and the user. The implemented sensors collect detailed information in real time from the environment to the user to deliver it to the remote caregiver. The integrated camera captures the actual image showing the user's field of vision. Other essential raw data such as GPS coordinates and step detector data make it easier and better to locate the user for the remote caregiver.

The leds are implanted in the frame of the glasses and can blink in different combinations to provide navigation commands. The position of the indicators on the frame is shown in Fig. 4 to provide different combinations of the

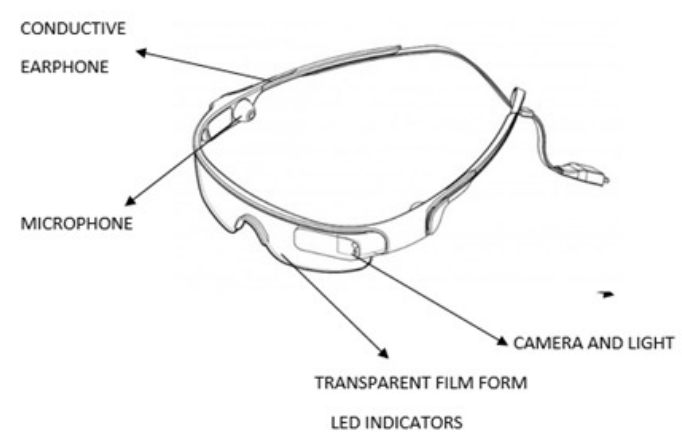

Fig. 3. Design of the Electronic Glasses

blinking indicator, in which seven indicators are implanted in the frame of each lens of the glasses. These indicators can blink in up to three different colors that come in red, green and finally yellow so that they generate navigation commands based on the traffic light image. The indicator combinations are integrated with a different blink pattern and are designed to transmit expressive navigation signals. Usability experiments evaluate the efficiency of these visual signals and qualify the different combinations in conjunction with patterns. It should be noted that the combination means that several indicators at once with an integrated pattern comes to make a group of adjustments by the frequency of blinking with the intensity of light [22].

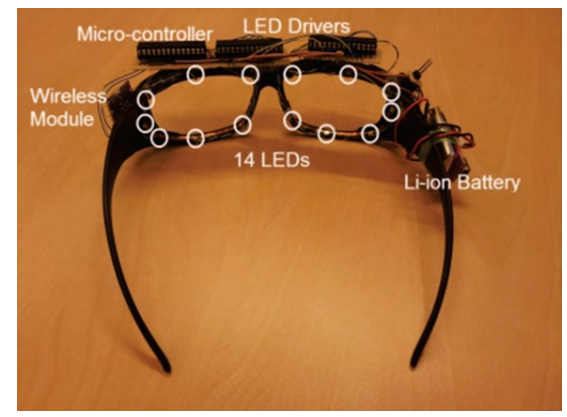

Fig. 4. LED Indicators Implanted in the Frame of the Glasses

In this study, an innovative concept is introduced to design a noble device to help elderly people with simple daily navigation tasks. The prototype of intelligent glasses based on indicators has been developed to provide users with visual signals that do not interfere with their vision of the physical environment. The diagram of the system and the integral components of the system are shown in Fig. 5. A lithiumion battery generates a stable $3.3 \mathrm{~V}$ and provides electrical power to all components. The Android application is developed to establish a command pattern and activate individual or combined indicators to flash. The application connects to the Bluetooth serial port profile (SPP) module on the device.

To realize the usability in the built prototype of the electronic glasses, two Android applications are developed. Two applications communicate with the device as described above. The difference is the user interface of the application to set 


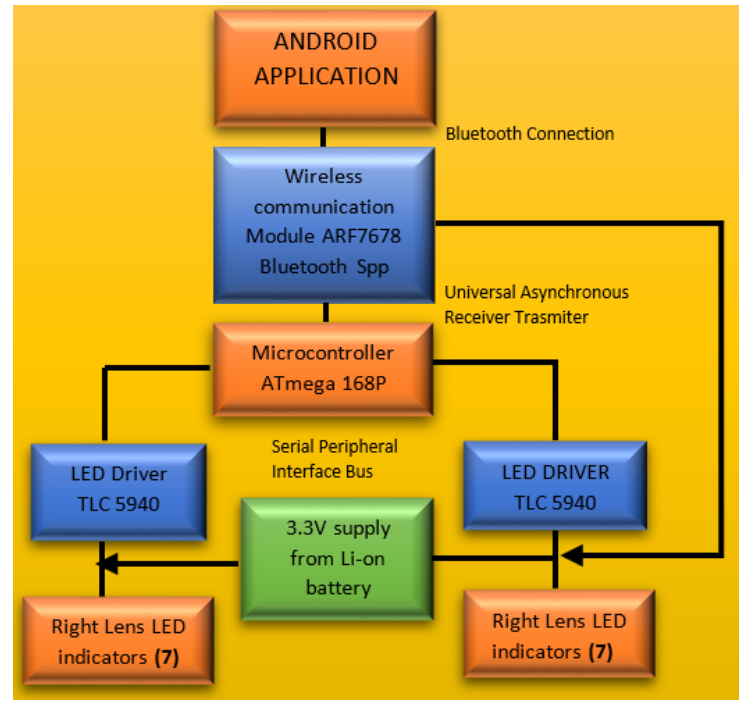

Fig. 5. Circuit Diagram Illustrates Component Connectivity

the blink pattern for individual indicators and the combination of indicators as navigation signals [23].In the first Android application, different indicators are set and sent to the device through the Bluetooth connection with colors, brightness and duration of the blink time. Fig. 6 shows the application's graphical user interface. In the second Android application, the navigation buttons are included in the graphical user interface on the other hand, Fig. 7 shows the user interface of the Android application to guide users in which specific visual signals can be assigned to each navigation button in the configuration menu [24].

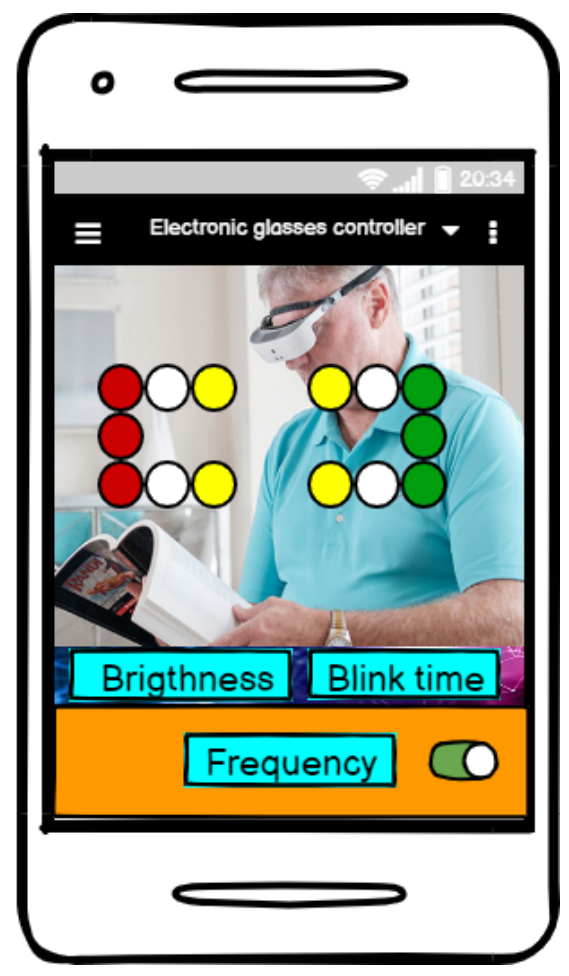

Fig. 6. Android Application

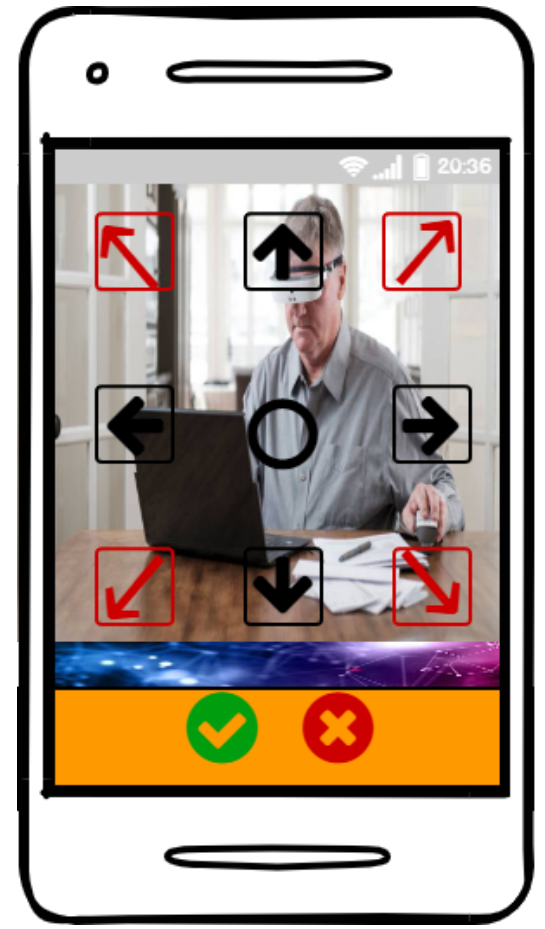

Fig. 7. Visual Commands

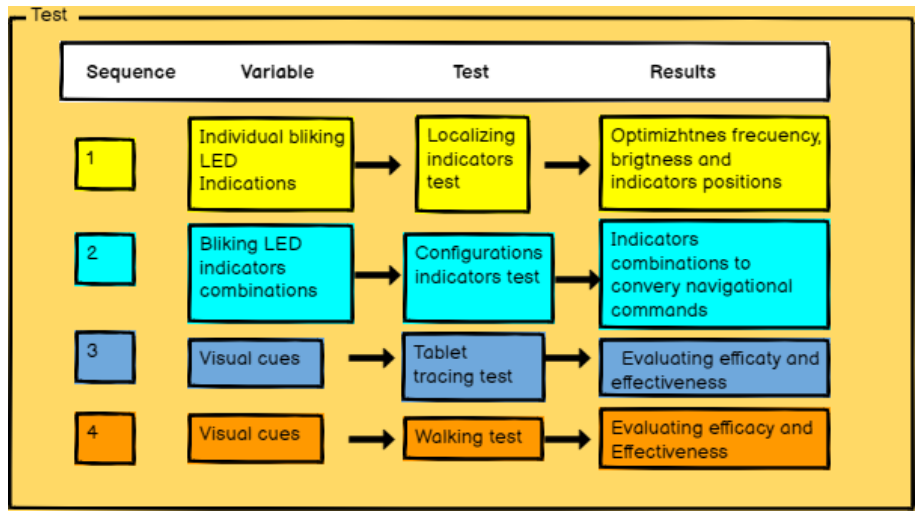

Fig. 8. Sequence of the Four Step Experiments

6) Test: In this last phase the usability of the prototype is tested, for real life situations in the users. We use the research method of design science with a qualitative and quantitative approach. Hevner et al.(2015) provided the guidance for the development of a design science artifact through iterative usability testing. The guide suggests early testing and usability experiments with real users, to evaluate and improve the system. For this purpose, we designed four-step experiments with different user groups [25].In Fig. 8, it shows the sequence of the experiments and specifies the factors and variables measured in the experiments. The first experiment is the location indicator test and aims to evaluate the visibility of the individual LEDs in the frame. The indicators are blinking individually. The result showed accuracy in recognizing individual blinking indicators for user display. In addition, the results clarify the satisfactory blinking pattern of the blinking indicators, including the optimized brightness and frequency. 


\section{Results AND Discussion}

\section{A. About the Case Study}

For the case study, the design of electronic glasses to help the elderly was carried out so that users obtain electronic glasses that allow them to capture images in high quality and in real time to improve visual perception and with it, daily autonomy. the objective is to facilitate the elderly in their daily activities, so the equipment is designed so that they continue to have both hands free and good mobility. Balsamiq Mockups was used as the first tool for the design of prototypes, since it is a tool that allows to design in a more effective and simple way that is used to make mobile applications and interfaces and thus be able to obtain an ideal prototype [26]. However, for the creation of the mobile application we also use Android Estudio, which is a software that offers different tools and services so that different users can create and innovate with new applications, starting from the code to the design of the user interface. This tool is very simple for that reason it facilitates the users to be able to understand the handling quickly and to be able to make the creation of our mobile application.

\section{B. About the Methodology}

Design Thinking is a model of how innovations in insecure environments can be approached in an agile and radical way. Design Thinking has a series of tools that are used throughout the process of creating innovative products and services, depending on the stage you are at. Design Thinking can always be used because it is based on problem solving from the user's perspective [27]. The Design Thinking methodology was chosen because it is considered very suitable for proposing IT solutions in process models where prototypes are used to clarify the requirements to the satisfaction of the user or client. Some of these process models are used as prototypes for their solution. They allow a lot of interaction with users such as the creation and evaluation of the prototypes created.

\section{- Advantages}

One of the advantages of using this Design Thinking methodology is that it encourages the immediate solution of problems that may arise. It is for this reason that the user or client is placed as the center for the creation process, in which the development is clear to overcome challenges by providing guidance on the techniques and tools to be used to solve a challenge both in a company and in other environments. It is necessary to emphasize that this methodology is focused on people, this means that it is a customer-centered method whose important potential is to solve real problems that people face. Adding another advantage to this is that it focuses on adapting the solution to the product and service on the needs of the user in that sense you get good results.

- Disadvantages

The disadvantage of the Design Thinking methodology is that it cannot be used for all types of projects.

- Comparison

Design Thinking is not only used by organizations or en- trepreneurs, in such a way that large and prestigious companies have discovered its benefits and have incorporated them into their daily work. Design Thinking places clients as the main focus for the creation process as opposed to other approaches that try to move from thought to action. Design thinking invites the consumer to assume a more active role in the design of the required product, therefore, it implies that the agents are in dialogue between the client and the developer. The first approach of a team in innovation, it is important to make it in a guided way and focused on a specific problem to apply the Design Thinking methodology in this way a process is proposed where the phases can be taken up again and repeated without major restrictions, while in Scrum, you can work with a constant flow of projects that must respond to the priorities of the business, this is how there is a series of events or meetings to follow in each Sprint. This is where there can be a contrast, however, some elements can be taken from Scrum such as prioritization, or organizational aspects can be adopted that contribute to teamwork.

\section{CONClusions}

In conclusion, the design of the electronic glasses will help the elderly to be able to comfortably perform the usual tasks, thanks to these electronic glasses the elderly will no longer escape from their reality and will not have any interruption or external stimulus that will prevent them from enjoying this new technological experience. On the other hand, the use of the Design Thinking methodology was fundamental, since it is focused on the user and the problems that may arise, which made the development of the prototype design and application possible. With this design of electronic glasses, it is intended in the future to have the implementation of the software in order to be able to implement it in Peru in that way giving a better quality of life to the elderly since the use of electronic devices is increasingly accepted, for the benefit of elderly patients with low vision, allowing people with partial visual disability to see more clearly.

\section{REFERENCES}

[1] R. Peleg-Adler, J. Lanir, and M. Korman, "The effects of aging on the use of handheld augmented reality in a route planning task," Computers in Human Behavior, vol. 81, pp. 52-62, 2018.

[2] M. L. Bianco, S. Pedell, and G. Renda, "Augmented reality and home modifications: a tool to empower older adults in fall prevention," pp. 499-507, 2016.

[3] S. Gopalakrishnan, S. C. Suwalal, G. Bhaskaran, R. Raman et al., "Use of augmented reality technology for improving visual acuity of individuals with low vision," Indian Journal of Ophthalmology, vol. 68, no. 6, p. 1136, 2020.

[4] H. P. van der Aa, H. C. Comijs, B. W. Penninx, G. H. van Rens, and R. M. van Nispen, "Major depressive and anxiety disorders in visually impaired older adults," Investigative ophthalmology \& visual science, vol. 56, no. 2, pp. 849-854, 2015.

[5] E. R. Vieira, F. Civitella, J. Carreno, M. G. Junior, C. F. Amorim, N. D’Souza, E. Ozer, F. Ortega, and J. A. Estrázulas, "Using augmented reality with older adults in the community to select design features for an age-friendly park: A pilot study," Journal of Aging Research, vol. 2020, 2020.

[6] C. Y. Huang, J. B. Thomas, A. Alismail, A. Cohen, W. Almutairi, N. S. Daher, M. H. Terry, and L. D. Tan, "The use of augmented reality glasses in central line simulation:"see one, simulate many, do one competently, and teach everyone"," Advances in medical education and practice, vol. 9, p. 357, 2018. 
[7] M. Ibarra Yancan de Villegas and J. Montes Pariona, "Efectividad del programa educativo "salud ocular" en el autocuidado en cuidadores del adulto mayor postoperado de catarata en el instituto nacional de oftalmología 2018," 2018.

[8] Y. Zhao, M. Hu, S. Hashash, and S. Azenkot, "Understanding low vision people's visual perception on commercial augmented reality glasses," in Proceedings of the 2017 CHI Conference on Human Factors in Computing Systems, 2017, pp. 4170-4181.

[9] F. Mostajeran, F. Steinicke, O. J. Ariza Nunez, D. Gatsios, and D. Fotiadis, "Augmented reality for older adults: Exploring acceptability of virtual coaches for home-based balance training in an aging population," in Proceedings of the 2020 CHI Conference on Human Factors in Computing Systems, 2020, pp. 1-12.

[10] C. Wrigley, E. Nusem, and K. Straker, "Implementing design thinking: Understanding organizational conditions," California Management Review, vol. 62, no. 2, pp. 125-143, 2020.

[11] K. Gurusamy, N. Srinivasaraghavan, and S. Adikari, "An integrated framework for design thinking and agile methods for digital transformation," in Design, User Experience, and Usability: Design Thinking and Methods, A. Marcus, Ed. Cham: Springer International Publishing, 2016, pp. 34-42.

[12] T. Fonseca Borges, L. M. Gil Morales, and Y. Ruiz Vega, "Impacto social de la atenciA visual en personas geritricas," Conrado, vol. 15, pp. 425 - 428, 122019.

[13] J. Ramke, A. B. Zwi, A. Palagyi, I. Blignault, and C. E. Gilbert, "Equity and blindness: closing evidence gaps to support universal eye health," Ophthalmic epidemiology, vol. 22, no. 5, pp. 297-307, 2015.

[14] G. T. Liu, N. J. Volpe, and S. L. Galetta, "3 - visual loss: Overview, visual field testing, and topical diagnosis," in Liu, Volpe, and Galetta's Neuro-Ophthalmology (Third Edition), third edition ed., G. T. Liu, N. J. Volpe, and S. L. Galetta, Eds. Elsevier, 2019, pp. 39 - 52.

[15] C. Creuzot-Garcher, C. Binquet, S. Daniel, L. Bretillon, N. Acar, A. de Lazzer, L. Arnould, C. Tzourio, A. M. Bron, and C. Delcourt, "The montrachet study: study design, methodology and analysis of visual acuity and refractive errors in an elderly population," Acta ophthalmologica, vol. 94, no. 2, pp. e90-e97, 2016.

[16] I. V. Malov, Y. V. Bantsykina, V. M. Malov, E. B. Eroshevskaya, and O. V. Pavlova, "Cataract problems in patients with high myopia (clinical case)," Aspirantskiy Vestnik Povolzhiya, vol. 19, no. 5-6, pp. 67-71, 2019.
[17] R. Ratnapriya, O. A. Sosina, M. R. Starostik, M. Kwicklis, R. J. Kapphahn, L. G. Fritsche, A. Walton, M. Arvanitis, L. Gieser, A. Pietraszkiewicz et al., "Retinal transcriptome and eqtl analyses identify genes associated with age-related macular degeneration," Nature genetics, vol. 51, no. 4, pp. 606-610, 2019.

[18] J. I. Orlando, H. Fu, J. B. Breda, K. van Keer, D. R. Bathula, A. DiazPinto, R. Fang, P.-A. Heng, J. Kim, J. Lee et al., "Refuge challenge: A unified framework for evaluating automated methods for glaucoma assessment from fundus photographs," Medical image analysis, vol. 59, p. $101570,2020$.

[19] F. De Marchi, L. Corrado, E. Bersano, M. Sarnelli, V. Solara, S. D'Alfonso, R. Cantello, and L. Mazzini, "Ptosis and bulbar onset: an unusual phenotype of familial als?" Neurological Sciences, vol. 39, no. 2, pp. 377-378, 2018.

[20] J. Yang, "Composition for the prevention and/or improvement of dry eye syndrome, dryness of the eye, and stiff neck and shoulder," May 14 2020, uS Patent App. 16/740,843.

[21] T. Hagos, M. Zechner, J. DiMarzio, and R. Green, "What's in an android application," in Beginning Android Games Development. Springer, 2020, pp. 45-54.

[22] Y. Kashimoto, A. Firouzian, Z. Asghar, G. Yamamoto, and P. Pulli, "Twinkle megane: Near-eye led indicators on glasses in tele-guidance for elderly," in 2016 IEEE International Conference on Pervasive Computing and Communication Workshops (PerCom Workshops). IEEE, 2016, pp. 1-6.

[23] S. K. Rao, R. K. Rao, and R. K. Rao, "Image and augmented reality based networks using mobile devices and intelligent electronic glasses," Jun. 28 2016, uS Patent 9,380,177.

[24] S. Machida and S. Kouji, "Control device for variable focus lenses, control method for variable focus lenses, and electronic glasses," Jan. 10 2017, uS Patent 9,541,774.

[25] W. Diyatmika, J. P. Chu, B. T. Kacha, C.-C. Yu, and C.-M. Lee, "Thin film metallic glasses in optoelectronic, magnetic, and electronic applications: a recent update," Current Opinion in Solid State and Materials Science, vol. 19, no. 2, pp. 95-106, 2015.

[26] H. Plattner, C. Meinel, and L. Leifer, Design thinking research: Taking breakthrough innovation home. Springer, 2016.

[27] E. Knight, J. Daymond, and S. Paroutis, "Design-led strategy: how to bring design thinking into the art of strategic management," California Management Review, vol. 62, no. 2, pp. 30-52, 2020. 